\title{
Enhanced Interval Analysis for Phase Stability: Cubic Equation of State Models
}

\author{
James Z. Hua* \\ Department of Chemical Engineering \\ University of Illinois at Urbana-Champaign \\ Urbana, IL 61801 USA \\ Joan F. Brennecke \\ and \\ Mark A. Stadtherr ${ }^{\dagger}$ \\ Department of Chemical Engineering \\ University of Notre Dame \\ Notre Dame, IN 46556 USA
}

July 1997

(revised January 1998)

${ }^{*}$ currently at Shell Chemical Company, Houston, TX

${ }^{\dagger}$ Author to whom all correspondence should be addressed. Phone: (219)631-9318; Fax: (219)631-8366;

E-mail: markst@nd.edu 


\begin{abstract}
The reliable prediction of phase stability is a challenging computational problem in chemical process simulation, optimization and design. The phase stability problem can be formulated either as a minimization problem or as an equivalent nonlinear equation solving problem. Conventional solution methods are initialization dependent, and may fail by converging to trivial or non-physical solutions or to a point that is a local but not global minimum. Thus there has been considerable recent interest in developing more reliable techniques for stability analysis. Recently we have demonstrated, using cubic equation of state models, a technique that can solve the phase stability problem with complete reliability. The technique, which is based on interval analysis, is initialization independent, and if properly implemented provides a mathematical guarantee that the correct solution to the phase stability problem has been found. However, there is much room for improvement in the computational efficiency of the technique. In this paper we consider two means of enhancing the efficiency of the method, both based on sharpening the range of interval function evaluations. Results indicate that by using the enhanced method, computation times can be reduced by nearly an order of magnitude in some cases.
\end{abstract}




\section{Introduction}

The determination of phase stability, i.e., whether or not a given mixture can split into multiple phases, is a key step in phase equilibrium calculations, and thus in the simulation and design of a wide variety of processes, especially those involving separation operations such as distillation and extraction. The phase stability problem is frequently formulated in terms of the tangent plane condition (Baker et al., 1982). Minima in the tangent plane distance are sought, usually by solving a system of nonlinear equations for the stationary points (Michelsen, 1982). If any of these yield a negative tangent plane distance, indicating that the tangent plane intersects (or lies above) the Gibbs energy of mixing surface, the phase is unstable and can split (in this context, unstable refers to both the metastable and classically unstable cases). The difficulty lies in that, in general, given any arbitrary equation of state or activity coefficient model, most computational methods cannot find with complete certainty all the stationary points, and thus there is no guarantee that the phase stability problem has been correctly solved.

Standard methods (e.g., Michelsen, 1982) for solving the phase stability problem typically rely on the use of multiple initial guesses, carefully chosen in an attempt to locate all stationary points in the tangent plane distance function. However, these methods offer no guarantee that the global minimum in the tangent plane distance has been found. Because of the difficulties that thus arise, there has been significant recent interest in the development of more reliable methods for solving the phase stability problem (e.g., Nagarajan et al., 1991; Sun and Seider, 1995; Eubank et al., 1992; Wasylkiewicz et al., 1996; McDonald and Floudas, 1995a,b,c,1997). For example, Sun and Seider

(1995) apply a homotopy-continuation method, which will often find all the stationary points, and is easier to initialize than Michelsen's approach. However, their technique is still initialization de- 
pendent and provides no theoretical guarantees that all stationary points have been found. The "area" method of Eubank et al. (1992), which is based on exhaustive search over a grid, can also be very reliable. They suggest that a course grid be used first to find the approximate location of solutions. Then, regions appearing not to contain a solution are eliminated from consideration and the search continues with a finer grid in the remaining regions. However, there is no mathematical guarantee provided that the regions eliminated do not contain solutions. McDonald and Floudas (1995a,b,c,1997) show that for certain activity coefficient models, the phase stability problem can be reformulated to make it amenable to solution by powerful global optimization techniques, generally involving branch and bound using convex underestimating functions. This method does provide a mathematical guarantee that the global minimum of the tangent plane distance has been found. While global optimization methods based on branch and bound can provide mathematical guarantees, in principle this guarantee can be lost computationally if the technique is implemented in floating point arithmetic, due to rounding error. As shown by the example given originally by Rump (1988) and also discussed by Hansen (1992), the impact of rounding error is something that should not be taken lightly. However, if the variables are well scaled, which is not the case in Rump's example, but which occurs naturally in this application when compositions are expressed as mole fractions, then rounding errors are unlikely to cause difficulties in practice. Nevertheless, in principle, rounding errors can accumulate even in well scaled problems.

An alternative approach for solving the phase stability problem, based on interval analysis, that provides both mathematical and computational guarantees of global optimality, with resolution limited only by machine precision, was originally suggested by Stadtherr et al. (1995), who applied it in connection with activity coefficient models, as later done also by McKinnon et al. (1996). This technique, in particular the use of an interval Newton/generalized bisection algorithm, is 
initialization independent and can solve the phase stability problem with mathematical certainty, while also dealing automatically with rounding error. Recently Hua et al. (1996a,b) extended this method to problems modeled with cubic equation of state (EOS) models, in particular the Van der Waals (VDW), Peng-Robinson (PR) and Soave-Redlich-Kwong (SRK) models with standard mixing rules. Though the technique developed is general-purpose, the applications presented here focus on these models as well.

While the interval approach can provide guarantees of reliability, there remains much room for improvement in the computational efficiency of the technique. In this paper we consider two means of enhancing the efficiency of the method, both based on sharpening the range of interval function evaluations. Results indicate that by using the enhanced method, computation times can be reduced by nearly an order of magnitude in some cases.

\section{Background}

\subsection{Phase Stability Analysis}

The determination of phase stability is often done using tangent plane analysis (Baker et al., 1982; Michelsen, 1982). A phase at specified temperature $T$, pressure $P$, and feed mole fraction $\mathbf{z}$ is unstable if the molar Gibbs energy of mixing surface $m(\mathbf{x}, v)=\Delta g_{m i x}=\Delta \hat{G}_{m i x} / R T$ ever falls below a plane tangent to the surface at $\mathbf{z}$. That is, if the tangent plane distance

$$
D(\mathbf{x}, v)=m(\mathbf{x}, v)-m_{0}-\sum_{i=1}^{n}\left(\frac{\partial m}{\partial x_{i}}\right)_{0}\left(x_{i}-z_{i}\right)
$$

is negative for any composition $\mathbf{x}$, the phase is unstable. The subscript zero indicates evaluation at $\mathbf{x}=\mathbf{z}, n$ is the number of components, and $v$ is the molar volume of the mixture. A common approach for determining if $D$ is ever negative is to minimize $D$ subject to the mole fractions 
summing to one and subject to the equation of state relating $\mathbf{x}$ and $v$. It is readily shown that the stationary points in this optimization problem can be found by solving the system of nonlinear equations:

$$
\begin{gathered}
{\left[\left(\frac{\partial m}{\partial x_{i}}\right)-\left(\frac{\partial m}{\partial x_{n}}\right)\right]-\left[\left(\frac{\partial m}{\partial x_{i}}\right)-\left(\frac{\partial m}{\partial x_{n}}\right)\right]_{0}=0, \quad i=1, \ldots, n-1} \\
1-\sum_{i=1}^{n} x_{i}=0 \\
P-\frac{R T}{v-b}+\frac{a}{v^{2}+u b v+w b^{2}}=0
\end{gathered}
$$

Equation (4) is the generalized cubic EOS given by Reid et al. (1987). With the appropriate choice of $u$ and $w$, common models such as PR $(u=2, w=-1)$, SRK $(u=1, w=0)$, and VDW $(u=0, w=0)$ may be obtained. For all the example problems considered here, standard mixing rules, namely $b=\sum_{i=1}^{n} x_{i} b_{i}$ and $a=\sum_{i=1}^{n} \sum_{j=1}^{n} x_{i} x_{j} a_{i j}$, are used, with $a_{i j}=\left(1-k_{i j}\right) \sqrt{a_{i i} a_{j j}}$. The $a_{i i}(T)$ and $b_{i}$ are pure component properties determined from the system temperature $T$, the critical temperatures $T_{c i}$, the critical pressures $P_{c i}$ and acentric factors $\omega_{i}$. If there are multiple real volume roots at the feed composition $\mathbf{z}$, then in evaluating equations (2) and (3), the molar volume $v_{0}$ at the feed composition must be the root yielding the minimum value of $m_{0}=m\left(\mathbf{z}, v_{0}\right)$, the reduced molar Gibbs energy of mixing at the feed.

The $(n+1) \times(n+1)$ system given by equations $(2)-(4)$ above has a trivial root at $(\mathbf{x}, v)=\left(\mathbf{z}, v_{0}\right)$ and frequently has multiple nontrivial roots as well. Thus conventional equation solving techniques may fail by converging to the trivial root or give an incorrect answer to the phase stability problem by converging to a stationary point that is not the global minimum of $D$. This is aptly demonstrated by the experiments of Green et al. (1993), who show that the pattern of convergence from different initial guesses demonstrates a complex fractal-like behavior for even very simple models like VDW. 
We use here an interval Newton/generalized bisection method for solving the system of equations (2)-(4). The method requires no initial guess, and will find with certainty enclosures of all the stationary points of the tangent plane distance $D$.

\subsection{Interval Computations}

A real interval, $X$, is defined as the continuum of real numbers lying between (and including) given upper and lower bounds; i.e., $X=[a, b]=\{x \in \Re \mid a \leq x \leq b\}$, where $a, b \in \Re$ and $a \leq b$. A real interval vector $\mathbf{X}=\left(X_{i}\right)=\left(X_{1}, X_{2}, \ldots, X_{n}\right)^{T}$ has $n$ real interval components and since it can be interpreted geometrically as an $n$-dimensional rectangle, is frequently referred to as a box. Note that in this section lower case quantities are real numbers and upper case quantities are intervals. Several good introductions to computation with intervals are available, including monographs by Neumaier (1990), Hansen (1992), and Kearfott (1996).

Of particular interest here are interval Newton/generalized bisection (IN/GB) methods. These techniques provide the power to find, with confidence, enclosures of all solutions of a system of nonlinear equations (Neumaier, 1990; Kearfott and Novoa, 1990), and to find with total reliability the global minimum of a nonlinear objective function (Hansen, 1992), provided only that upper and lower bounds are available for all variables. Efficient techniques for implementing IN/GB are a relatively recent development, and thus such methods have not yet been widely applied. Schnepper and Stadtherr (1990) suggested the use of this method for solving chemical process modeling problems, and recently described an implementation (Schnepper and Stadtherr, 1996). Balaji et al. (1995) have also successfully applied the method to chemical engineering problems.

For a system of nonlinear equations $\mathbf{f}(\mathbf{x})=\mathbf{0}$ with $\mathbf{x} \in \mathbf{X}^{(0)}$, the basic iteration step in interval 
Newton methods is, given an interval $\mathbf{X}^{(k)}$, to solve the linear interval equation system

$$
F^{\prime}\left(\mathbf{X}^{(k)}\right)\left(\mathbf{N}^{(k)}-\mathbf{x}^{(k)}\right)=-\mathbf{f}\left(\mathbf{x}^{(k)}\right)
$$

for a new interval $\mathbf{N}^{(k)}$, where $k$ is an iteration counter, $F^{\prime}\left(\mathbf{X}^{(k)}\right)$ is an interval extension of the real Jacobian $f^{\prime}(\mathbf{x})$ of $f(\mathbf{x})$ over the current interval $\mathbf{X}^{(k)}$, and $\mathbf{x}^{(k)}$ is a point in the interior of $\mathbf{X}^{(k)}$. It can be shown (Moore, 1966) that any root $\mathbf{x}^{*} \in \mathbf{X}^{(k)}$ is also contained in the image $\mathbf{N}^{(k)}$, suggesting the iteration scheme $\mathbf{X}^{(k+1)}=\mathbf{X}^{(k)} \cap \mathbf{N}^{(k)}$. While this iteration scheme can be used to tightly enclose a solution, what is also of significance here is the power of equation (5) as an existence and uniqueness test. For several techniques for finding $\mathbf{N}^{(k)}$ from equation (5), it can be proven (e.g., Neumaier, 1990; Kearfott, 1996) that if $\mathbf{N}^{(k)} \subset \mathbf{X}^{(k)}$, then there is a unique zero of $\mathbf{f}(\mathbf{x})$ in $\mathbf{X}^{(k)}$, and that Newton's method with real arithmetic can be used to find it, starting from any point in $\mathbf{X}^{(k)}$. This suggests a root inclusion test for $\mathbf{X}^{(k)}$ :

1. (Range Test) Compute an interval extension $\mathbf{F}\left(\mathbf{X}^{(k)}\right)$ containing the range of $\mathbf{f}(\mathbf{x})$ over $\mathbf{X}^{(k)}$ and test to see whether it contains zero. Clearly, if $0 \notin \mathbf{F}\left(\mathbf{X}^{(k)}\right) \supseteq\left\{\mathbf{f}(\mathbf{x}) \mid \mathbf{x} \in \mathbf{X}^{(k)}\right\}$ then there can be no solution of $\mathbf{f}(\mathbf{x})=\mathbf{0}$ in $\mathbf{X}^{(k)}$ and this interval need not be further tested.

2. (Interval Newton Test) Compute the image $\mathbf{N}^{(k)}$ by solving equation (5).

(a) If $\mathbf{X}^{(k)} \cap \mathbf{N}^{(k)}=\emptyset$, then there is no root in $\mathbf{X}^{(k)}$.

(b) If $\mathbf{N}^{(k)} \subset \mathbf{X}^{(k)}$, then there is exactly one root in $\mathbf{X}^{(k)}$.

(c) If neither of the above is true, then no further conclusion can be drawn.

In the last case, one could then repeat the root inclusion test on the next interval Newton iterate $\mathbf{X}^{(k+1)}$, assuming it is sufficiently smaller than $\mathbf{X}^{(k)}$, or one could bisect $\mathbf{X}^{(k+1)}$ and repeat the root inclusion test on the resulting intervals. This is the basic idea of interval Newton/generalized 
bisection methods. A detailed step-by-step description of the basic IN/GB algorithm used here is given by Schnepper and Stadtherr (1996).

Once an enclosure has been found in 2(a) above that contains a unique root, that enclosure can be tightened by continuing the interval Newton iteration, which will converge quadratically. Alternatively, a point approximation of the root can be located using a (point-valued) Newton's method, which will converge to the root starting from any point in the enclosure. If desired, a verified enclosure for the root can then be obtained by doing an $\epsilon$-expansion (Kearfott, 1996) around the point approximation of the root to obtain a narrow enclosure, and then verifing that this contains the root using the range and interval Newton tests above. This latter procedure is what is used here.

Though it does not occur in any of the problems considered here, in some cases the bisection process may continue until an interval narrower than the prescribed precision is obtained that still satisfies the range test, but that does not satisfy the test $2(a)$ for containing a unique root. This typically occurs if there is a root at or very near a singular point. In this case, the interval may contain one or more roots. In the context of phase stability analysis, this does not present any difficulties since, as emphasized below, the underlying problem is a global minimization problem and it is thus not necessary to find all the stationary points.

The system of equations (2)-(4) to be solved here involves $n+1$ variables, the $n$ component mole fractions $\mathbf{x}$ and the molar volume $v$. For the mole fraction variables, initial intervals of $[0,1]$ are suitable. In practice the initial lower bound is set to an arbitrarily small positive number $\varepsilon$ $\left(10^{-10}\right.$ was used) to avoid taking the logarithm of zero in subsequent calculations. This can be done without the loss of reliability providing a sufficiently small value of $\varepsilon$ is used. The lower limit on the molar volume was taken to be the smallest pure component size parameter $b_{i}$, and the upper 
bound was taken to be the ideal gas molar volume for the $T$ and $P$ under investigation. Although it is possible to have compressibility factors greater than one at very high reduced pressure, this was deemed satisfactory for the range of reduced temperature and pressure explored below. For these problems, the initial upper bound on $v$ can be further increased without significant impact on computational efficiency since intervals with these higher values of volume tend to be quickly eliminated based on the range test for the EOS. Our implementation of the IN/GB method for the phase stability problem is based on appropriately modified double-precision routines from the packages INTBIS (Kearfott and Novoa, 1990) and INTLIB (Kearfott et al., 1994).

\section{Enhancements}

The efficiency of the method discussed above depends to a considerable extent on how tightly one can compute interval extensions $F(\mathbf{X})$ of real expressions $f(\mathbf{x})$. Denoting $F^{R}(\mathbf{X})=\{f(\mathbf{x}) \mid \mathbf{x} \in \mathbf{X}\}$ as the exact range of $f(\mathbf{x})$ over the interval $\mathbf{X}$, then an interval extension of $f(\mathbf{x})$ is an enclosure for $F^{R}$, that is, $F(\mathbf{X}) \supseteq F^{R}(\mathbf{X})$. The most common approach to enclosing $F^{R}$ is to use the natural interval extension of $f(\mathbf{x})$, which is obtained from the expression $f(\mathbf{x})$ by simply replacing each occurrence of the variable $\mathbf{x}$ by the interval $\mathbf{X}$ and evaluating the real arithmetic operations using the corresponding interval arithmetic operations (Moore, 1966). Computing the natural interval extension of an expression may generate its actual range $F^{R}$, but unfortunately it is not uncommon for the natural interval extension to provide a drastic overestimation for $F^{R}$. This can be seen, for example, using the simple expression $f(x)=x /(x-1)$ and evaluating its interval extension for $x \in[2,3]$. Computing the natural interval extension yields $F([2,3])=[2,3] /([2,3]-1)=$ $[2,3] /[1,2]=[1,3]$ which is a considerable overestimate of the true range $F^{R}([2,3])=[1.5,2]$. In general, such overestimations may occur when an interval variable appears more than once in an 
expression. This so-called "dependence problem" occurs because interval arithmetic essentially treats all occurrences of a variable independently rather than recognizing their dependence. For example, if one rearranges $f(x)=x /(x-1)$ so that $x$ appears only once, $f(x)=1+1 /(x-1)$, then the natural interval extension yields $F([2,3])=1+1 /([2,3]-1)=1+1 /[1,2]=1+[0.5,1]=[1.5,2]$, which the same as $F^{R}$. In fact, it can be shown that if each variable in an expression appears only once that then the natural interval extension will always yield the true (though, in computational practice, outwardly rounded) range $F^{R}$. Unfortunately for most of the functions of interest in phase stability analysis it is not possible to perform rearrangements that eliminate all but one occurrence of each variable. Thus we need to consider other means of computing tighter interval extensions.

There have been considerable efforts made toward developing systematic methods for most sharply bounding the range of a given real function over an interval (e.g., Ratschek and Rokne, 1984; Rokne, 1986; Neumaier, 1990). Among these are the use of centered forms of the function, such as the mean value form, and the use of slope forms. We concentrate here on two other approaches, namely the use of monotonicity (e.g. Hansen, 1992) and the use of constraint information. Both are relatively simple and inexpensive computationally, and offer the potential to obtain the true range.

\subsection{Monotonicity}

If a function is known to be monotonic on a given interval, then clearly its range can be tightly bounded simply by evaluating the function at the endpoints of the interval. For a function $f(x)$ of a single variable, if $f(x)$ is known to be monotonically nondecreasing on the interval $X=[a, b]$, then its monotonic interval extension $F^{M}=[f(a), f(b)]$ yields the true range $F^{R}$. Similarly, if $f(x)$ is monotonically nonincreasing on $X$, then $F^{M}=[f(b), f(a)]=F^{R}$. To determine whether or not 
$f(x)$ is monotonic, the natural interval extension $F^{\prime}$ of its first derivative $f^{\prime}=\partial f / \partial x$ can be used. Thus if $F^{\prime}(X) \geq 0$ then $f$ is known to be monotonically nondecreasing on $X$ and if $F^{\prime}(X) \leq 0$ then $f$ is known to be monotonically nonincreasing on $X$. Testing for and using monotonicity will frequently yield sharper bounds than the natural interval extension. For example, consider $f(x)=x \ln x$ on the interval $X=[0.4,0.5]$. The natural interval extension yields $F=[0.4,0.5] \times$ $\ln [0.4,0.5]=[0.4,0.5] \times[-0.9163,-0.6932]=[-0.4582,-0.2772]$. Checking for monotonicity, it is seen that $f^{\prime}(x)=1+\ln x$ and $F^{\prime}=1+\ln [0.4,0.5]=[0.0837,0.3069] \geq 0$, and thus $F^{M}=$ $[0.4 \ln 0.4,0.5 \ln 0.5]=[-0.3666,-0.3465]$, which is only about $10 \%$ the width of the natural interval extension, and which is (within roundout) the true range $F^{R}$. The monotonic interval extension $F^{M}$ for a function of more than one variable is also easily computed, as explained in detail by Hansen (1992). If for every variable the function is either monotonically nonincreasing or monotonically nondecreasing, then $F^{M}=F^{R}$; otherwise, $F^{M}$ is no less sharp than the natural extension. The effect of using monotonicity in evaluating interval extensions in the context of the phase stability problem is shown below in Section 4.

\subsection{Mole Fraction Weighted Averages}

Mole fraction weighted averages of pure component property parameters appear frequently in the set of equations (2)-(4) used for phase stability analysis. If these expressions can be more sharply bounded it will lead to sharper bounds on the functions in which they appear. Thus, we develop here an interval extension for mole fraction weighted averages. Consider the mole fraction weighted average $f(\mathbf{x})=\bar{s}=\sum_{i=1}^{n} x_{i} s_{i}$, where the $s_{i}$ are constants and $\sum_{i=1}^{n} x_{i}=1$. The natural interval extension of $\bar{s}$ will yield the true range of the expression in the space in which all the mole fraction variables $x_{i}$ are independent. However, the range can be tightened by considering 
the constraint that the mole fractions must sum to one. Thus we seek to compute the constrained space interval extension $F^{C S}$ of $f(\mathbf{x})=\bar{s}$.

One approach for doing this is simply to eliminate one of the mole fraction variables, say $x_{n}$. Then an enclosure for the range of $\bar{s}$ in the constrained space can be determined by computing the natural interval extension of $s_{n}+\sum_{i=1}^{n-1}\left(s_{i}-s_{n}\right) x_{i}$. However, as we will see below, this may not yield the sharpest possible bounds on $\bar{s}$ in the constrained space. For the mole fraction interval $\mathbf{X}=\left[\mathbf{x}^{L}, \mathbf{x}^{U}\right]=\left(X_{i}\right)$, with $X_{i}=\left[x_{i}^{L}, x_{i}^{U}\right]$, the true upper and lower bounds on $\bar{s}$ in the constrained space are solutions of the linear programming (LP) problem

$$
\text { opt } \bar{s}=\sum_{i=1}^{n} x_{i} s_{i}
$$

subject to

$$
\begin{gathered}
1-\sum_{i=1}^{n} x_{i}=0 \\
x_{i}^{L} \leq x_{i} \leq x_{i}^{U}, \quad i=1, \ldots, n .
\end{gathered}
$$

This is a standard LP problem with $N=3 n$ variables, the $n$ mole fractions plus $n$ slack variables and $n$ surplus variables, and with $M=2 n+1$ constraints, the normality condition plus $n$ upper bounds and $n$ lower bounds. At an optimal vertex of an LP problem, there are $N-M=n-1$ variables (the nonbasic variables) that must equal zero. Since in general the $x_{i}^{L}$ are nonzero and thus the $x_{i}$ are nonzero, this indicates that $n-1$ of the slack or surplus variables must be zero. Thus, at the minimum or maximum of $\bar{s}$ in the constrained space, at least $n-1$ of the mole fraction variables must be at their upper or lower bound. This knowledge can be used (Tessier, 1997) to easily construct the desired upper and lower bounds for $F^{C S}$ without actually using an LP problem solver.

To do this we note that, using the natural interval extension, the lower bound on $\bar{s}$ is evaluated 
as $\bar{s}\left(\mathbf{x}^{L}\right)$, and $\sum_{i=1}^{n} x_{i}^{L} \leq 1$ (if not then there is no point in $\mathbf{X}$ that can satisfy equation (3) and so this interval can be discarded). To evaluate the lower bound of $F^{C S}=\left[\bar{s}^{L}, \bar{s}^{U}\right]$, we want to increase some components of $\mathbf{x}^{L}$ to a point $\mathbf{x}^{-}$at which $\sum_{i=1}^{n} x_{i}^{-}=1$, and to do this in such a way that $\bar{s}\left(\mathbf{x}^{-}\right)$will be the best lower bound $\bar{s}^{L}$ of $\bar{s}$. This can be done by first increasing as much as possible (considering the interval bounds) the mole fraction $x_{i}$ for which the corresponding $s_{i}$ is the lowest, then repeating for the next lowest, continuing until $\sum_{i=1}^{n} x_{i}^{-}=1$. Similarly, to evaluate the upper bound of $F^{C S}$, we want to decrease some components of $\mathbf{x}^{U}$ to a point $\mathbf{x}^{+}$at which $\sum_{i=1}^{n} x_{i}^{+}=1$, and to do this in such a way that $\bar{s}\left(\mathbf{x}^{+}\right)$will be the best upper bound $\bar{s}^{U}$ of $\bar{s}$. This can be done by first decreasing as much as possible (considering the interval bounds) the mole fraction $x_{i}$ for which $s_{i}$ is the lowest, then repeating for the next lowest, continuing until $\sum_{i=1}^{n} x_{i}^{+}=1$.

Algorithmically, this can be expressed as follows:

1. Rank the $s_{i}$ and let $r_{k}$ be the index $i$ of the $s_{i}$ ranked $k$-th from lowest. For example, if $n=4$ and $s_{3} \leq s_{4} \leq s_{1} \leq s_{2}$, then $r_{1}=3, r_{2}=4, r_{3}=1$ and $r_{4}=2$.

2. Initialize $\mathbf{x}^{-}=\mathbf{x}^{L}$ and $\mathbf{x}^{+}=\mathbf{x}^{U}$. Also, define $\sigma^{-}=\sum_{i=1}^{n} x_{i}^{-}$and $\sigma^{+}=\sum_{i=1}^{n} x_{i}^{+}$. If $\sigma^{-}>1$ or $\sigma^{+}<1$, then eliminate this interval and stop.

3. Determine $\mathbf{x}^{-}$. For $k=1: n$,

(a) If $\sigma^{-}+\left(x_{r_{k}}^{U}-x_{r_{k}}^{L}\right) \leq 1$, then set $x_{r_{k}}^{-}=x_{r_{k}}^{U}$ and update $\sigma^{-}$.

(b) Else set $x_{r_{k}}^{-}=x_{r_{k}}^{L}+\left(1-\sigma^{-}\right)$. Return current $\mathbf{x}^{-}$and proceed to determine $\mathbf{x}^{+}$in the next step.

4. Determine $\mathbf{x}^{+}$. For $k=1: n$,

(a) If $\sigma^{+}-\left(x_{r_{k}}^{U}-x_{r_{k}}^{L}\right) \geq 1$, then set $x_{r_{k}}^{+}=x_{r_{k}}^{L}$ and update $\sigma^{+}$. 
(b) Else set $x_{r_{k}}^{+}=x_{r_{k}}^{U}-\left(\sigma^{+}-1\right)$. Return current $\mathbf{x}^{+}$and proceed to determine $\bar{s}^{L}$ and $\bar{s}^{U}$ in the next step.

5. Compute $\bar{s}^{L}=\bar{s}\left(\mathbf{x}^{-}\right)$and $\bar{s}^{U}=\bar{s}\left(\mathbf{x}^{+}\right)$.

Note that at $\mathbf{x}^{-}$and $\mathbf{x}^{+}$all but one of the components, the one determined in step 3(b) for $\mathbf{x}^{-}$and in step 4(b) for $\mathbf{x}^{+}$are at their upper or lower bounds. In general, the one remaining component will not be at a bound, but it may be. In practice, the above algorithm is implemented in interval arithmetic.

For example, consider the case $n=3$ with $s_{1}=1, s_{2}=2$ and $s_{3}=3$ on the mole fraction interval $X_{1}=[0.1,0.4], X_{2}=[0.2,0.4]$ and $X_{3}=[0.3,0.5]$. The natural interval extension of $\bar{s}=x_{1}+2 x_{2}+3 x_{3}$ is $[0.1+2(0.2)+3(0.3), 0.4+2(0.4)+3(0.5)]=[1.4,2.7]$. Using the approach in which $x_{n}=x_{3}$ is eliminated, we would instead compute the natural interval extension of $3-2 x_{1}-x_{2}$, which is $3-[0.2,0.8]-[0.2,0.4]=[2.2,2.8]-[0.2,0.4]=[1.8,2.6]$. While this is an improvement over the unconstrained natural extension, it is still does not represent the true range of $\bar{s}$ in the constrained space (since the range of $x_{3}$ is never used). To get the true range $F^{C S}$ we first determine $\mathbf{x}^{-}$as above. Initially $\sigma^{-}=0.6$ and $\mathbf{x}^{-}=\mathbf{x}^{L}=(0.1,0.2,0.3)^{\mathrm{T}}$. For $k=1, r_{k}=1$, and in step $3(\mathrm{a}), \sigma^{-}+\left(x_{1}^{U}-x_{1}^{L}\right)=0.6+(0.4-0.1)=0.9 \leq 1$, so we set $x_{1}^{-}=x_{1}^{U}=0.4$ and $\sigma^{-}=0.9$. For $k=2, r_{k}=2$, and in step $3(\mathrm{a}), \sigma^{-}+\left(x_{2}^{U}-x_{2}^{L}\right)=0.9+(0.4-0.2)=1.1 \not \leq 1$, so we set $x_{2}^{-}=x_{2}^{L}+\left(1-\sigma^{-}\right)=0.2+(1-0.9)=0.3$ and return $\mathbf{x}^{-}=(0.4,0.3,0.3)^{\mathrm{T}}$. Similarly, in step $4, \mathbf{x}^{+}$is determined to be $(0.1,0.4,0.5)^{\mathrm{T}}$. Then, $\bar{s}^{L}=\bar{s}\left(\mathbf{x}^{-}\right)=0.4+2(0.3)+3(0.3)=1.9$ and $\bar{s}^{U}=\bar{s}\left(\mathbf{x}^{+}\right)=0.1+2(0.4)+3(0.5)=2.4$. Thus, the constrained space extension is $F^{C S}=[1.9,2.4]$ and this is the tightest possible range for $\bar{s}$ in the constrained space.

For phase stability analysis, the constrained space interval extension can be used to evaluate 
the range of $b=\sum_{i=1}^{n} x_{i} b_{i}$ and of $a=\sum_{i=1}^{n} \sum_{j=1}^{n} x_{i} x_{j} a_{i j}$. Obtaining $F^{C S}$ for $b$ is done as described above. For $a$, we note that $a=\sum_{i=1}^{n} x_{i} \sum_{j=1}^{n} x_{j} a_{i j}=\sum_{i=1}^{n} x_{i} \bar{a}_{i}$, and first obtain the constrained space extension $\left[\bar{a}_{i}^{L}, \bar{a}_{i}^{U}\right]$ for each mole fraction weighted average $\bar{a}_{i}$ using the procedure above. We now want the range of $\sum_{i=1}^{n} x_{i}\left[\bar{a}_{i}^{L}, \bar{a}_{i}^{U}\right]=\sum_{i=1}^{n}\left[x_{i} \bar{a}_{i}^{L}, x_{i} \bar{a}_{i}^{U}\right]=\left[\sum_{i=1}^{n} x_{i} \bar{a}_{i}^{L}, \sum_{i=1}^{n} x_{i} \bar{a}_{i}^{U}\right]$, and note these upper and lower bounds are themselves mole fraction weighted averages. Thus, to get the constrained space interval extension of $a$ we get its lower bound by using the procedure above to determine the lower bound of $\sum_{i=1}^{n} x_{i} \bar{a}_{i}^{L}$ and its upper bound by using the procedure above to determine the upper bound of $\sum_{i=1}^{n} x_{i} \bar{a}_{i}^{U}$.

In the next section we consider several example problems and examine the extent to which the use of the constrained space and monotonic interval extensions improves computational efficiency.

\section{Results}

To test the enhancements in computational efficiency expected to be provided by using the constrained space and monotonic interval extensions for solving phase stability problems, as well as to verify that its reliability has not been affected, several different mixtures modeled by an equation of state (VDW, SRK or PR) have been used. In order to emphasize the power of the method, in these studies we have found all the stationary points. However, it should be emphasized that, for making a determination of phase stability or instability, finding all the stationary points is not always necessary nor desirable, as discussed in more detail below.

The results presented in Tables 1-8 below include roots (stationary points) found, as well as the value of the tangent plane distance $D$ at each root. It should be noted that, while point approximations are reported here, we have actually determined verified enclosures of each root and computed 
$D$ for this enclosure. Each such enclosure is known to contain a unique root, based on the intervalNewton uniqueness test described above. Thus, for example, in Table 1 , for the $z_{1}=0.0115$ feed, the $x_{1}$ value found for the third root was actually $x_{1}=[0.03258313704886784,0.03258313704886790]$ and the tangent plane distance is $D=[.01303899885308101, .01303899885315651]$. Similarly narrow enclosures were determined in all cases.

\subsection{Problem 1}

This is a mixture of hydrogen sulfide(1) and methane(2) at $190 \mathrm{~K}$ and $40.53 \mathrm{bar}$ (40 atm.). The SRK model was used with parameters calculated from $T_{c 1}=373.2 \mathrm{~K}, P_{c 1}=89.4$ bar, $\omega_{1}=0.1$, $T_{c 2}=190.6 \mathrm{~K}, P_{c 2}=46.0 \mathrm{bar}, \omega_{2}=0.008$, and a binary interaction parameter $k_{12}=0.08$.

Several feeds were considered, as shown in Table 1 . For the $z_{1}=0.5$ case, our results are consistent with those given by Sun and Seider (1995) for this problem. For the $z_{1}=0.0187$ case, it is well known, for feeds near this point, that this is a difficult problem to solve (e.g., Michelsen, 1982; Sun and Seider, 1995; Hua et al., 1996b). As noted by Michelsen and others, if one uses a locally convergent solver, with nearly pure $\mathrm{CH}_{4}$ as the initial guess, convergence will likely be to the trivial solution at $x_{1}=z_{1}=0.0187$. And if nearly pure $\mathrm{H}_{2} \mathrm{~S}$ is the initial guess, convergence will likely be to the local, but not global, minimum at $x_{1}=0.8848$. Using only these initial guesses would lead to the incorrect conclusion that the mixture is stable. This is indicative of the importance of the initialization strategy when conventional methods are used. An important advantage of the IN/GB approach described here is that it eliminates the initialization problem, since it is initialization independent. In this case, it finds all the stationary points, including the global minimum at $x_{1}=0.0767$, correctly predicting, since $D<0$ at this point, that a mixture with this feed composition is unstable (there will be a vapor-liquid split, with a methane-rich liquid 
phase). Michelsen's algorithm, as implemented in LNGFLASH from the IVC-SEP package (Hytoft and Gani, 1996), a code that in general we have found to be extremely reliable, incorrectly predicts that this mixture is stable. As indicated in Table 1, several other feed compositions were tested using the IN/GB approach, with correct results obtained in each case. It should be noted that the presence of multiple real volume roots in this problem does not present any difficulty, since the solver simply finds enclosures of all roots for the given system. Thus, nothing needs to be done to select the right volume roots (or compressibility factors).

Also included in Table 1 are the number of root inclusion tests performed in the computation and the total CPU time on a Sun Ultra 1/170 workstation. This is done for the case $(F)$ in which just the natural interval extensions are used, the case $\left(F^{C S}\right)$ in which the constrained space interval extension is used, and the case $\left(F^{C S M}\right)$ in which both the constrained space and monotonic interval extensions are used. In the latter case, there is a dramatic reduction in the number of intervals that must be tested for roots, and the computation time is reduced by an average of more than $80 \%$. Most of the savings, about a factor of four, can be attributed to the use of the constrained space interval extension to bound $a$ and $b$. We would still expect standard approaches to the phase stability problem to be faster, but those methods do not reliably solve the problem in all cases. Thus, as one might expect, to obtain guaranteed reliability some premium must be paid in terms of computation time.

\subsection{Problem 2}

This is a mixture of methane(1) and propane(2) at $277.6 \mathrm{~K}$; pressures of 50 bar and 100 bar were considered. The SRK model was used with parameters calculated from the methane pure component properties given above, $T_{c 2}=369.8 \mathrm{~K}, P_{c 2}=42.5 \mathrm{bar}, \omega_{2}=0.152$, and a binary 
interaction parameter $k_{12}=0.029$. Results are shown in Table 2 for $P=50$ bar and in Table 3 for $P=100$ bar, and are consistent with calculations shown in Prausnitz et al. (1986). The feeds of $z_{1}=0.68$ and 0.73 at 100 bar represent particularly difficult problems because they are in a region near the critical point of the mixture. In both cases, the interval method correctly predicts instability, as indicated by the negative value of $D$ for at least one stationary point. Again the use of the enhanced interval extensions provides significant computational savings, with about a $65-85 \%$ reduction in CPU time.

\subsection{Problem 3}

This is a binary mixture modeled using VDW and exhibiting type I behavior according to the classification of van Konynenburg and Scott (1980). Component 1 is carbon dioxide $\left(T_{c}=304.2\right.$ $\mathrm{K}, P_{c}=73.8 \mathrm{bar}$ ), and the properties of component 2 were chosen to replicate the example used by van Konynenburg and Scott for a Type I mixture (this corresponds to their values of the dimensionless parameters $\xi=0, \zeta=0.5$ and $\Lambda=-0.05)$. The resulting size and energy parameters are $b_{1}=b_{2}=42.8374 \mathrm{~cm}^{3} / \mathrm{mol}, a_{11}=3.6565 \times 10^{6}\left(\mathrm{~cm}^{6} \mathrm{bar}\right) / \mathrm{mol}^{2}, a_{22}=1.097 \times 10^{7}\left(\mathrm{~cm}^{6} \mathrm{bar}\right) / \mathrm{mol}^{2}$ and $a_{12}=7.6792 \times 10^{6}\left(\mathrm{~cm}^{6} \mathrm{bar}\right) / \mathrm{mol}^{2}$. The system is described in more detail by Hua et al. (1996a). Table 4 shows results for selected feeds at two different sets of conditions (reduced by the critical properties of $\mathrm{CO}_{2}$ ). In all cases the correct results are obtained. The use of the constrained space and monotonic interval extensions again yields substantial improvements in CPU time, with about a $65-75 \%$ reduction.

\subsection{Problem 4}

This is the ternary mixture used by Green et al. (1993) to demonstrate the wide regions of 
initial guesses for which a standard Newton's method will not converge, or converges to trivial or non-physical solutions. It is modeled using VDW with $b_{11}=b_{22}=b_{33}=61.5375 \mathrm{~cm}^{3} / \mathrm{mol}$, $a_{11}=5.11 \times 10^{6}\left(\mathrm{~cm}^{6} \mathrm{~atm}\right) / \mathrm{mol}^{2}, a_{22}=1.19 \times 10^{7}\left(\mathrm{~cm}^{6} \mathrm{~atm}\right) / \mathrm{mol}^{2}, a_{33}=8.52 \times 10^{6}\left(\mathrm{~cm}^{6} \mathrm{~atm}\right) / \mathrm{mol}^{2}$, $a_{12}=2 a_{11}, a_{13}=\left(a_{11} a_{33}\right)^{1 / 2}$, and $a_{23}=\left(a_{22} a_{33}\right)^{1 / 2}$. Four feeds at $P=80$ atm. and $T=400 K$ were considered. The results are shown in Table 5 and match those of Green et al. (1993). Using both the constrained space and monotonic interval extensions gives about a $75 \%$ reduction in CPU time on these problems, with again most of this obtained as a result of the constrained space extension.

\subsection{Problem 5}

This is a mixture of nitrogen(1) and ethane(2) at $270 \mathrm{~K}$ and 76 bar. The PR model was used with parameters calculated from $T_{c 1}=126.2 \mathrm{~K}, P_{c 1}=33.9 \mathrm{bar}, \omega_{1}=0.04, T_{c 2}=305.4 \mathrm{~K}, P_{c 2}$ $=48.8$ bar, $\omega_{2}=0.098$, and a binary interaction parameter $k_{12}=0.08$. Results for several feed compositions are shown in Table 6, and are consistent with results in Prausnitz et al. (1986). Several other values of $T, P$, and $z_{1}$ have also been tried for this system and correct results were obtained. Use of the enhanced interval extensions for computing the range of $a$ and $b$ results in computation time savings of around $70 \%$ on these problems.

\subsection{Problem 6}

This is a mixture of carbon dioxide(1) and methane(2) at $220 \mathrm{~K}$ and 60.8 bar. The PR model was used with parameters calculated from $T_{c 1}=304.2 \mathrm{~K}, P_{c 1}=73.8 \mathrm{bar}, \omega_{1}=0.225$, the methane parameters given above, and a binary interaction parameter $k_{12}=0.095$. Results for several feed compositions are shown in Table 7, and are consistent with results in Prausnitz et al. (1986). 
Several other values of $T, P$, and $z_{1}$ have also been tried for this system and correct results were obtained. The improvement in computational efficiency due to using the enhanced interval extensions is substantial, with a reduction in computing time of around 80-85\% when both the constrained space and monotonic extensions are used.

\section{7 $\quad$ Problem 7}

This is a mixture of nitrogen(1), methane(2) and ethane(3) at $270 \mathrm{~K}$ and 76 bar. The PR model was used with the pure component parameters given above, and the binary interaction parameters $k_{12}=0.038, k_{13}=0.08$ and $k_{23}=0.021$. Four feeds were considered, with results shown in Table 8. The first two feeds are not stable and the other two feeds are stable. Again, these results are consistent with results in Prausnitz et al. (1986). The second and third feeds represent particularly difficult problems, since they are in the vicinity of the critical point of the mixture, yet the interval algorithm has no problem determining the correct solutions. For these two problems, use of the enhanced interval extensions yields a reduction in computing time of about $88 \%$, nearly an order of magnitude savings. Savings on the other two feed compositions are impressive as well.

\subsection{Discussion}

The results clearly show that substantial computational savings can be obtained by using the enhanced interval extensions described above. Most of the improvements, a factor of over five on the most difficult problems, can be attributed to the constrained space extension used to more tightly bound the $a$ and $b$ parameters in the EOS model. This in turn leads to tighter bounds on expressions containing these parameters. Further tightening of bounds can then be obtained using the monotonic interval extension. The tightening of bounds on function ranges allows intervals 
to be eliminated more readily than if bounds are not sharp. For instance, if the true range $F^{R}$ of a function over an interval does not contain zero, then that interval can be eliminated from further consideration, but an overestimate of $F^{R}$, such as might be obtained from the natural interval extension, might well contain zero, leading to further work on this interval and perhaps its bisection, creating even more work. The use of the monotonic and constrained space interval extension represent an attempt to prevent this by obtaining bounds that more tightly enclose the true range.

While we have focused here on improving the interval function evaluations that underly the IN/GB approach for phase stability analysis, we are currently implementing several other simple ways in which its efficiency can be improved (Hua et al., 1997). For example, in the problems above we used IN/GB to find enclosures of all the stationary points. However, for making a determination of phase stability or instability, finding all the stationary points is not always necessary. For example if an interval is encountered over which the interval evaluation of $D$ has a negative upper bound, this guarantees that there is a point at which $D<0$, and so one can immediately conclude that the mixture is unstable without determining all the stationary points. It is also possible to make use of the underlying global minimization problem. Since the objective function $D$ has a known value of zero at the mixture feed composition, any interval over which the interval value of $D$ has a lower bound greater than zero cannot contain the global minimum and can be discarded, even though it may contain a stationary point (at which $D$ will be positive and thus not of interest). Thus, we can essentially combine the interval-Newton technique with an interval branch and bound procedure in which lower bounds are generated using interval techniques as opposed to convex underestimators. Also, it should be noted that the method described here can easily be combined with existing local methods for determining phase stability. First, the (fast) local method is used. If it indicates 
instability then this is the correct answer as it means a point at which $D<0$ has been found. If the local method indicates stability, however, this may not be the correct answer since the local method may have missed the global minimum in $D$. Applying the new method described here can then be used to confirm that the mixture is stable if that is the case, or to correctly determine that it is really unstable if that is the case (Hua et al., 1997).

These techniques for improving the efficiency of the method, together with the work described here on tightening interval extensions, will be particularly important as problems involving a larger number of components are considered. Like any technique (e.g., branch and bound) based on a binary tree, the worst case computational complexity of the method described is exponential. Our previous experience (Schnepper and Stadtherr, 1996) on larger problems indicates that computation times become unpredictable and that a larger number of variables does not necessarily imply a larger computation time. This is consistent with our preliminary work on four and five component problems, which shows that computation times for finding all stationary points vary widely from a few seconds to tens of minutes. Fortunately, however, implementing the ideas described in this section, and not finding all stationary points can reduce computation times by orders of magnitude in many cases.

\section{Conclusions and Significance}

Results on several problems, involving VDW, SRK and PR EOS models of binary and ternary mixtures, indicate that the enhancements described here can substantially improve the efficiency of the interval Newton/general bisection approach for phase stability analysis, providing significant ( $>65 \%$ computational savings on all problems and savings of nearly an order of magnitude on some. The interval Newton/generalized bisection algorithm can solve phase stability problems 
for a generalized cubic equation of state model efficiently and with complete reliability, providing a method that can guarantee with mathematical certainty that the correct result is found, and thus eliminating computational problems that are frequently encountered with currently available techniques. The method is initialization independent; it is also model independent, straightforward to use, and can be applied in connection with other equations of state or with activity coefficient models.

Acknowledgments - This work has been supported in part by the donors of The Petroleum Research Fund, administered by the ACS, under Grant 30421-AC9, by the National Science Foundation Grants CTS95-22835 and DMI96-96110, by the Environmental Protection Agency Grant R824731-01-0, by the Department of Energy Grant DE-FG07-96ER14691, and by a grant from Sun Microsystems, Inc. We also acknowledge Mr. Gang Xu for his assistance in revising this paper. 


\section{References}

Baker, L. E.; Pierce, A. C.; Luks, K. D. Gibbs energy analysis of phase equilibria. Soc. Petrol. Engrs. J. 1982, 22, 731-742.

Balaji, G. V.; Seader, J. D. Application of interval-Newton method to chemical engineering problems. AIChE Symp. Ser 1995, $91(304), 364-367$.

Eubank, A. C.; Elhassan, A. E.; Barrufet, M. A.; Whiting, W. B. Area method for prediction of fluid-phase equilibria. Ind. Eng. Chem. Res. 1992, 31, 942-949.

Green, K. A.; Zhou, S.; Luks, K. D. The fractal response of robust solution techniques to the stationary point problem. Fluid Phase Equilibria 1993, 84, 49-78.

Hansen, E. R. Global Optimization Using Interval Analysis. Marcel Dekkar, Inc.: New York, 1992.

Hansen, E. R.; Sengupta, R. I. Bounding solutions of systems of equations using interval analysis. BIT 1981, 21, 203-211.

Hua, J. Z.; Brennecke, J. F.; Stadtherr, M. A. Combined local and global approach to reliable computation of phase equilibria. Presented at AIChE Annual Meeting, Paper 80b, Los Angeles, CA, November, 1997.

Hua, J. Z.; Brennecke, J. F.; Stadtherr, M. A. Reliable prediction of phase stability using an interval-Newton method. Fluid Phase Equilibria 1996a, 116, 52-59.

Hua, J. Z.; Brennecke, J. F.; Stadtherr, M. A. Reliable phase stability analysis for cubic equation of state models. Comput. Chem. Eng. 1996b, 20, S395-S400. 
Hytoft, G.; Gani, R. IVC-SEP Program Package, Tech. Rep. SEP 8623. Institut for Kemiteknik, Danmarks Tekniske Universitet: Lyngby, Denmark, 1996.

Kearfott, R. B. Interval arithmetic techniques in the computational solution of nonlinear systems of equations: Introduction, examples, and comparisons. Lectures in Applied Mathematics 1990, $26,337-357$.

Kearfott, R. B. Rigorous Global Search: Continuous Problems. Kluwer Academic Publishers: Dordrecht, 1996.

Kearfott, R. B.; Dawande, M.; Du, K.-S.; Hu, C.-Y. Algorithm 737: INTLIB, A portable FORTRAN 77 interval standard function library. ACM Trans. Math. Software 1994, 20, 447-459.

Kearfott, R. B.; Novoa, M. Algorithm 681: INTBIS, A portable interval Newton/bisection package. ACM Trans. Math. Software 1990, 16, 152-157.

McDonald, C. M.; Floudas, C. A. Global optimization for the phase stability problem. AIChE J. 1995a, 41, 1798-1814.

McDonald, C. M.; Floudas, C. A. Global optimization for the phase and chemical equilibrium problem: Application to the NRTL equation. Comput. Chem. Eng. 1995b, 19, 1111-1139.

McDonald, C. M.; Floudas, C. A. Global optimization and analysis for the Gibbs free energy function using the UNIFAC, Wilson, and ASOG Equations. Ind. Eng. Chem. Res. 1995c, 34, $1674-1687$.

McDonald, C. M.; Floudas, C. A. GLOPEQ: A new computational tool for the phase and chemical equilibrium problem. Comput. Chem. Eng. 1997, 21, 1-23. 
McKinnon, K. I. M.; Millar, C. G.; Mongeau, M. Global optimization for the chemical and phase equilibrium problem using interval analysis. In State of the Art in Global Optimization: Computational Methods and Applications; Floudas, C. A.; Pardalos, P. M., Eds.; Kluwer Academic Publishers, 1996, pp. 365-382.

Michelsen, M. L. The isothermal flash problem. Part I: Stability. Fluid Phase Equilibria 1982, 9, $1-19$.

Moore, R. E. Interval Analysis. Prentice-Hall: Englewood Cliffs, NJ, 1966.

Nagarajan, N. R.; Cullick, A. S.; Griewank, A. New strategy for phase equilibrium and critical point calculations by thermodynamic energy analysis. Part I. Stability analysis and flash. Fluid Phase Equilibria 1991, 62, 191-210.

Neumaier, A. Interval Methods for Systems of Equations. Cambridge University Press: Cambridge, England, 1990.

Prausnitz, J. M.; Lichtenthaler, R. N.; Gomes de Azevedo, E. Molecular Thermodynamics of FluidPhase Equilibria. Prentice-Hall: Englewood Cliffs, NJ, 1986.

Ratschek, H.; Rokne, J. Computer Methods for the Range of Functions. Halsted Press: New York, 1984.

Reid, R. C.; Prausnitz, J. M.; Poling, B. E. The Properties of Gases and Liquids.. McGraw-Hill: New York, 1987.

Rokne, J. G. Low complexity $k$-dimensional centered forms. Computing 1986, 37, 247-253. 
Rump, S. M. Algorithm for verified inclusions - theory and practice. In Reliability in Computing; Moore, R. E., ed.; San Diego: Academic Press, 1988 pp. 109-126.

Schnepper, C. A.; Stadtherr, M. A. On using parallel processing techniques in chemical process design. Presented at AIChE Annual Meeting, Chicago, November, 1990.

Schnepper, C. A.; Stadtherr, M. A. Robust process simulation using interval methods. Comput. Chem. Eng. 1996, 20, 187-199.

Stadtherr, M. A.; Schnepper, C. A.; Brennecke, J. F. Robust phase stability analysis using interval methods. AIChE Symp. Ser. 1995, $91(304), 356-359$.

Sun, A. C.; Seider, W. D. Homotopy-continuation method for stability analysis in the global minimization of the Gibbs free energy. Fluid Phase Equilibria 1995, 103, 213-249.

Tessier, S. R. Enhanced Interval Analysis for Phase Stability: Excess Gibbs Energy Models. Master's thesis, University of Notre Dame, Notre Dame, IN, 1997.

van Konynenburg, P. H.; Scott, R. L. Critical lines and phase equilibria in binary van der Waals mixtures. Philos. Trans. R. Soc. London, Ser. A 1980, 298, 495-540.

Wasylkiewicz, S. K.; Sridhar, L. N.; Malone, M. F.; Doherty, M. F. Global stability analysis and calculation of liquid-liquid equilibrium in multicomponent mixtures. Ind. Eng. Chem. Res. 1996, 35, 1395-1408. 


\section{Table Headings}

Table 1. Problem 1: SRK, hydrogen sulfide(1) and methane(2) at $P=40.53$ bar and $T=190$ K. Comparison of using natural interval extensions $(F)$, constrained space interval extensions $\left(F^{C S}\right)$, and constrained space plus monotonic interval extensions $\left(F^{C S M}\right)$.

Table 2. Problem 2: SRK, methane(1) and propane(2) at $P=50$ bar and $T=277.6 \mathrm{~K}$.

Table 3. Problem 2: SRK, methane (1) and propane (2) at $P=100$ bar and $T=277.6 \mathrm{~K}$.

Table 4. Problem 3: VDW, binary type I mixture.

Table 5. Problem 4: VDW, ternary mixture of Green et al. (1993), $P=80$ atm, $T=400 \mathrm{~K}$.

Table 6. Problem 5: PR, nitrogen(1) and ethane(2) at $P=76$ bar and $T=270 \mathrm{~K}$.

Table 7. Problem 6: PR, carbon dioxide(1) and methane(2) at $P=60.8$ bar and $T=220 \mathrm{~K}$.

Table 8. Problem 7: PR, nitrogen(1), methane(2) and ethane(3), $P=76$ Bar, $T=270 \mathrm{~K}$. 
Table 1: Problem 1: SRK, hydrogen sulfide(1) and methane(2) at $P=40.53$ bar and $T=190 \mathrm{~K}$. Comparison of using natural interval extensions $(F)$, constrained space interval extensions $\left(F^{C S}\right)$, and constrained space plus monotonic interval extensions $\left(F^{C S M}\right)$.

\begin{tabular}{|c|c|c|c|c|c|c|c|c|}
\hline \multirow{2}{*}{$\begin{array}{c}\text { Feed } \\
\left(z_{1}, z_{2}\right)\end{array}$} & \multirow{2}{*}{$\begin{array}{c}\text { Roots } \\
\left(x_{1}, x_{2}, v\right)\end{array}$} & \multirow[t]{2}{*}{ D } & \multicolumn{3}{|c|}{$\begin{array}{c}\text { Number of Root } \\
\text { Inclusion Tests }\end{array}$} & \multicolumn{3}{|c|}{$\begin{array}{l}\text { CPU Time (sec) } \\
\text { Sun Ultra } 1 / 170\end{array}$} \\
\hline & & & $F$ & $F^{C S}$ & $F^{C S M}$ & $F$ & $F^{C S}$ & $F^{C S M}$ \\
\hline$(0.0115,0.9885)$ & $\begin{array}{l}(0.0115,0.9885,212.8) \\
(0.0237,0.9763,97.82) \\
(0.0326,0.9674,78.02)\end{array}$ & $\begin{array}{c}0.0 \\
0.0137 \\
0.0130\end{array}$ & 5424 & 1385 & 1079 & 1.024 & 0.298 & 0.234 \\
\hline$(0.0187,0.9813)$ & $\begin{array}{l}(0.8848,0.1152,36.58) \\
(0.0187,0.9813,207.3) \\
(0.0313,0.9687,115.4) \\
(0.0767,0.9233,64.06) \\
(0.4905,0.5095,41.50)\end{array}$ & $\begin{array}{c}0.0109 \\
0.0 \\
0.0079 \\
-0.004 \\
0.0729\end{array}$ & 8438 & 1928 & 1428 & 1.671 & 0.416 & 0.290 \\
\hline$(0.07,0.93)$ & $\begin{array}{c}(0.8743,0.1257,36.65) \\
(0.5228,0.4772,40.89) \\
(0.0178,0.9822,208.0) \\
(0.0304,0.9696,113.7) \\
(0.07,0.93,65.35)\end{array}$ & $\begin{array}{c}0.0512 \\
0.0965 \\
0.0015 \\
0.0100 \\
0.0\end{array}$ & 8504 & 1927 & 1414 & 1.690 & 0.416 & 0.298 \\
\hline$(0.50,0.50)$ & $\begin{array}{c}(0.8819,0.1181,36.60) \\
(0.0184,0.9816,207.5) \\
(0.0311,0.9689,114.9) \\
(0.0746,0.9254,64.44) \\
(0.50,0.50,41.32)\end{array}$ & $\begin{array}{c}-0.057 \\
-0.079 \\
-0.071 \\
-0.082 \\
0.0\end{array}$ & 8406 & 1927 & 1416 & 1.660 & 0.416 & 0.291 \\
\hline$(0.888,0.112)$ & $\begin{array}{c}(0.888,0.112,36.55) \\
(0.0190,0.9810,207.1) \\
(0.0316,0.9684,116.0) \\
(0.0792,0.9208,63.60) \\
(0.4795,0.5205,41.72)\end{array}$ & $\begin{array}{c}0.0 \\
0.0026 \\
0.0103 \\
-0.002 \\
0.0683\end{array}$ & 8396 & 1918 & 1412 & 1.671 & 0.417 & 0.296 \\
\hline$(0.89,0.11)$ & $\begin{array}{c}(0.89,0.11,36.54) \\
(0.0192,0.9808,206.9) \\
(0.0319,0.9681,116.4) \\
(0.0809,0.9191,63.31) \\
(0.4725,0.5275,41.87)\end{array}$ & $\begin{array}{c}0.0 \\
0.0113 \\
0.0189 \\
0.0058 \\
0.0724 \\
\end{array}$ & 8410 & 1907 & 1411 & 1.673 & 0.421 & 0.294 \\
\hline
\end{tabular}


Table 2: Problem 2: SRK, methane(1) and propane(2) at $P=50$ bar and $T=277.6 \mathrm{~K}$.

\begin{tabular}{|c|c|c|c|c|c|c|c|c|}
\hline \multirow{2}{*}{$\begin{array}{l}\text { Feed } \\
\left(z_{1}, z_{2}\right)\end{array}$} & \multirow{2}{*}{$\begin{array}{c}\text { Roots } \\
\left(x_{1}, x_{2}, v\right)\end{array}$} & \multirow[t]{2}{*}{ D } & \multicolumn{3}{|c|}{$\begin{array}{l}\text { Number of Root } \\
\text { Inclusion Tests }\end{array}$} & \multicolumn{3}{|c|}{$\begin{array}{l}\text { CPU Time (sec) } \\
\text { Sun Ultra } 1 / 170\end{array}$} \\
\hline & & & $F$ & $F^{C S}$ & $F^{C S M}$ & $F$ & $F^{C S}$ & $F^{C S M}$ \\
\hline$(0.10,0.90)$ & $(0.10,0.90,86.71)$ & 0.0 & 1969 & 675 & 587 & 0.413 & 0.155 & 0.135 \\
\hline$(0.40,0.60)$ & $\begin{array}{c}(0.8654,0.1346,378.4) \\
(0.5515,0.4485,115.3) \\
(0.40,0.60,89.46)\end{array}$ & $\begin{array}{c}-0.153 \\
0.0106 \\
0.0\end{array}$ & 4345 & 1347 & 1179 & 0.905 & 0.318 & 0.260 \\
\hline$(0.60,0.40)$ & $\begin{array}{c}(0.7058,0.2942,313.0) \\
(0.60,0.40,216.5) \\
(0.1928,0.8072,86.07)\end{array}$ & $\begin{array}{c}-0.007 \\
0.0 \\
-0.223\end{array}$ & 3706 & 1210 & 1091 & 0.782 & 0.285 & 0.252 \\
\hline$(0.90,0.10)$ & $(0.90,0.10,388.5)$ & 0.0 & 3290 & 1023 & 878 & 0.640 & 0.216 & 0.181 \\
\hline
\end{tabular}


Table 3: Problem 2: SRK, methane (1) and propane (2) at $P=100$ bar and $T=277.6 \mathrm{~K}$.

\begin{tabular}{|c|c|c|c|c|c|c|c|c|}
\hline \multirow{2}{*}{$\begin{array}{l}\text { Feed } \\
\left(z_{1}, z_{2}\right)\end{array}$} & \multirow{2}{*}{$\begin{array}{c}\text { Roots } \\
\left(x_{1}, x_{2}, v\right)\end{array}$} & \multirow[t]{2}{*}{ D } & \multicolumn{3}{|c|}{$\begin{array}{l}\text { Number of Root } \\
\text { Inclusion Tests }\end{array}$} & \multicolumn{3}{|c|}{$\begin{array}{l}\text { CPU Time (sec) } \\
\text { Sun Ultra } 1 / 170\end{array}$} \\
\hline & & & $F$ & $F^{C S}$ & $F^{C S M}$ & $F$ & $F^{C S}$ & $F^{C S M}$ \\
\hline$(0.40,0.60)$ & $(0.40,0.60,82.22)$ & 0.0 & 2518 & 802 & 680 & 0.522 & 0.185 & 0.152 \\
\hline$(0.68,0.32)$ & $\begin{array}{c}(0.7721,0.2279,126.0) \\
(0.6881,0.3119,103.0) \\
(0.68,0.32,101.4)\end{array}$ & $\begin{array}{c}-3.3 \times 10^{-4} \\
4.10 \times 10^{-7} \\
0.0\end{array}$ & 19986 & 6266 & 3334 & 3.966 & 1.368 & 0.643 \\
\hline$(0.73,0.27)$ & $\begin{array}{c}(0.7567,0.2433,121.1) \\
(0.73,0.27,113.2) \\
(0.6506,0.3494,96.38)\end{array}$ & $\begin{array}{c}-2.0 \times 10^{-5} \\
0.0 \\
-2.9 \times 10^{-4}\end{array}$ & 14768 & 4588 & 2693 & 2.967 & 1.021 & 0.529 \\
\hline$(0.90,0.10)$ & $(0.90,0.10,165.2)$ & 0.0 & 2485 & 793 & 685 & 0.514 & 0.168 & 0.139 \\
\hline
\end{tabular}


Table 4: Problem 3: VDW, binary type I mixture.

\begin{tabular}{|c|c|c|c|c|c|c|c|c|}
\hline \multirow{2}{*}{$\begin{array}{c}\text { Feed } \\
\left(P_{r}, T_{r}, z_{1}, z_{2}\right)\end{array}$} & \multirow{2}{*}{$\begin{array}{c}\text { Roots } \\
\left(x_{1}, x_{2}, v\right)\end{array}$} & \multirow[t]{2}{*}{ D } & \multicolumn{3}{|c|}{$\begin{array}{l}\text { Number of Root } \\
\text { Inclusion Tests }\end{array}$} & \multicolumn{3}{|c|}{$\begin{array}{l}\text { CPU Time (sec) } \\
\text { Sun Ultra } 1 / 170\end{array}$} \\
\hline & & & $F$ & $F^{C S}$ & $F^{C S M}$ & $F$ & $F^{C S}$ & $F^{C S M}$ \\
\hline$(1.0,1.5,0.60,0.40)$ & $\begin{array}{c}(0.9472,0.0528,444.5) \\
(0.6791,0.3209,76.51) \\
(0.60,0.40,66.55)\end{array}$ & $\begin{array}{c}-0.3598 \\
0.00174 \\
0.0\end{array}$ & 4520 & 1853 & 1235 & 0.713 & 0.304 & 0.184 \\
\hline$(1.0,1.5,0.20,0.80)$ & $\begin{array}{c}(0.20,0.80,54.14) \\
(0.8101,0.1899,401.3) \\
(0.6577,0.3423,152.1)\end{array}$ & $\begin{array}{c}0.0 \\
0.3836 \\
0.4720\end{array}$ & 2303 & 873 & 751 & 0.398 & 0.152 & 0.126 \\
\hline$(1.0,1.5,0.95,0.05)$ & $(0.95,0.05,445.3)$ & 0.0 & 3738 & 1621 & 1083 & 0.618 & 0.245 & 0.153 \\
\hline$(3.24,2.0,0.40,0.60)$ & $(0.40,0.60,67.76)$ & 0.0 & 1785 & 859 & 681 & 0.319 & 0.146 & 0.108 \\
\hline
\end{tabular}




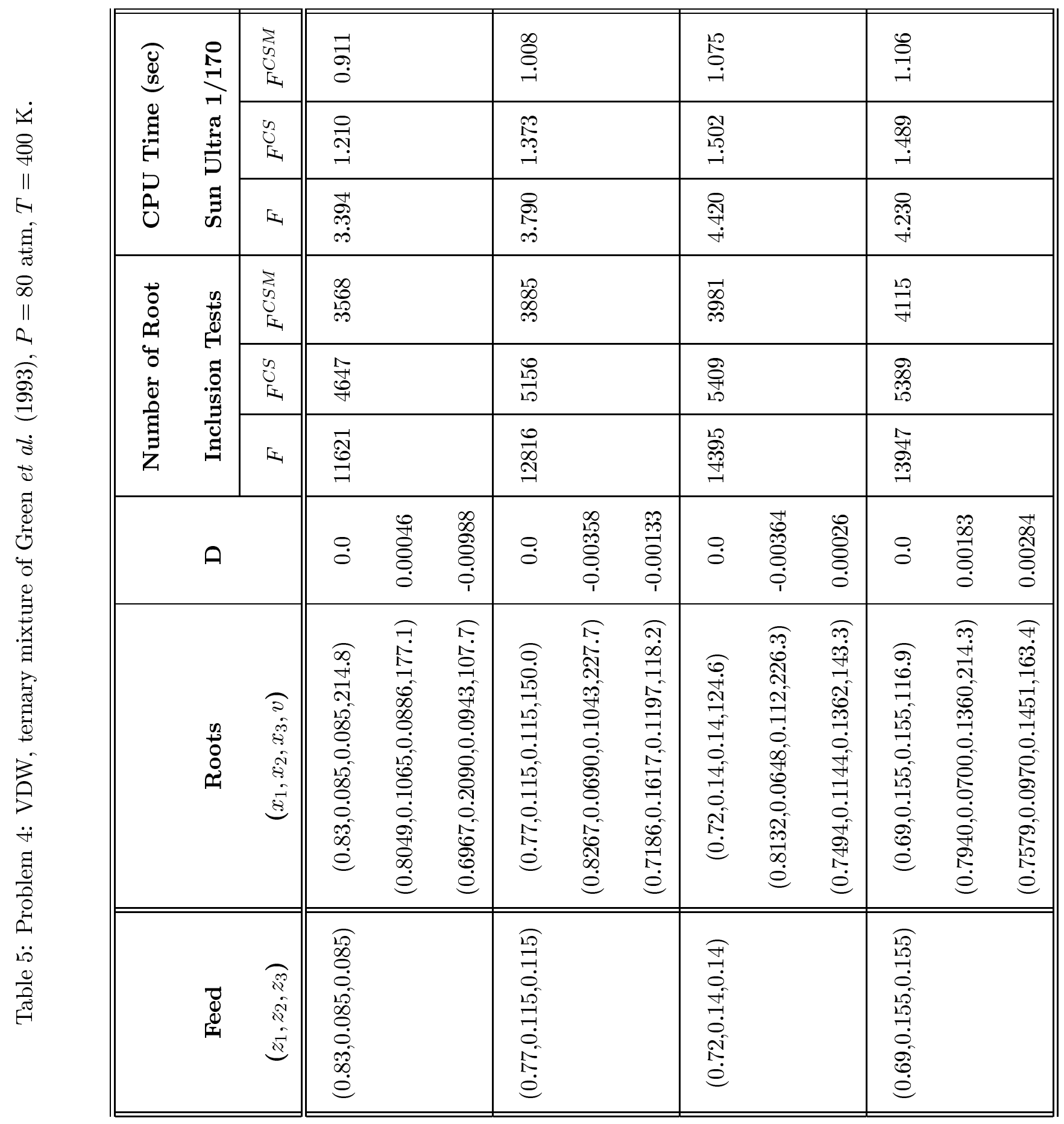


Table 6: Problem 5: PR, nitrogen(1) and ethane(2) at $P=76$ bar and $T=270 \mathrm{~K}$.

\begin{tabular}{|c|c|c|c|c|c|c|c|c|}
\hline \multirow{2}{*}{$\begin{array}{c}\text { Feed } \\
\left(z_{1}, z_{2}\right)\end{array}$} & \multirow{2}{*}{$\begin{array}{c}\text { Roots } \\
\left(x_{1}, x_{2}, v\right)\end{array}$} & \multirow[t]{2}{*}{ D } & \multicolumn{3}{|c|}{$\begin{array}{l}\text { Number of Root } \\
\text { Inclusion Tests }\end{array}$} & \multicolumn{3}{|c|}{$\begin{array}{l}\text { CPU Time (sec) } \\
\text { Sun Ultra } 1 / 170\end{array}$} \\
\hline & & & $F$ & $F^{C S}$ & $F^{C S M}$ & $F$ & $F^{C S}$ & $F^{C S M}$ \\
\hline$(0.10,0.90)$ & $(0.10,0.90,71.11)$ & 0.0 & 1881 & 630 & 604 & 0.400 & 0.144 & 0.134 \\
\hline$(0.18,0.82)$ & $\begin{array}{c}(0.4943,0.5057,198.3) \\
(0.2961,0.7039,110.4) \\
(0.18,0.82,78.61)\end{array}$ & $\begin{array}{c}-0.010 \\
0.0058 \\
0.0\end{array}$ & 4560 & 1444 & 1312 & 0.979 & 0.340 & 0.279 \\
\hline$(0.30,0.70)$ & $\begin{array}{c}(0.4893,0.5107,198.3) \\
(0.30,0.70,112.3) \\
(0.1767,0.8233,78.18)\end{array}$ & $\begin{array}{c}-0.0138 \\
0.0 \\
-0.007\end{array}$ & 4586 & 1446 & 1316 & 0.980 & 0.341 & 0.278 \\
\hline$(0.44,0.56)$ & $\begin{array}{c}(0.44,0.56,181.2) \\
(0.3353,0.6647,131.5) \\
(0.1547,0.8453,75.64)\end{array}$ & $\begin{array}{c}0.0 \\
0.0026 \\
-0.016\end{array}$ & 4649 & 1506 & 1293 & 0.991 & 0.359 & 0.279 \\
\hline$(0.60,0.40)$ & $(0.60,0.40,227.8)$ & 0.0 & 3312 & 947 & 901 & 0.651 & 0.201 & 0.178 \\
\hline
\end{tabular}


Table 7: Problem 6: PR, carbon dioxide(1) and methane(2) at $P=60.8$ bar and $T=220 \mathrm{~K}$.

\begin{tabular}{|c|c|c|c|c|c|c|c|c|}
\hline \multirow{2}{*}{$\begin{array}{c}\text { Feed } \\
\left(z_{1}, z_{2}\right)\end{array}$} & \multirow{2}{*}{$\begin{array}{c}\text { Roots } \\
\left(x_{1}, x_{2}, v\right)\end{array}$} & \multirow[t]{2}{*}{ D } & \multicolumn{3}{|c|}{$\begin{array}{l}\text { Number of Root } \\
\text { Inclusion Tests }\end{array}$} & \multicolumn{3}{|c|}{$\begin{array}{l}\text { CPU Time (sec) } \\
\text { Sun Ultra } 1 / 170\end{array}$} \\
\hline & & & $F$ & $F^{C S}$ & $F^{C S M}$ & $F$ & $F^{C S}$ & $F^{C S M}$ \\
\hline$(0.10,0.90)$ & $(0.10,0.90,168.5)$ & 0.0 & 4162 & 962 & 762 & 0.839 & 0.193 & 0.158 \\
\hline$(0.20,0.80)$ & $\begin{array}{c}(0.20,0.80,141.6) \\
(0.2589,0.7411,88.51) \\
(0.4972,0.5028,47.98)\end{array}$ & $\begin{array}{c}0.0 \\
0.0022 \\
-0.007\end{array}$ & 13630 & 3093 & 2141 & 2.827 & 0.713 & 0.484 \\
\hline$(0.30,0.70)$ & $\begin{array}{c}(0.1848,0.8152,141.6) \\
(0.30,0.70,69.79) \\
(0.3579,0.6421,59.13)\end{array}$ & $\begin{array}{c}-0.007 \\
0.0 \\
-1.9 \times 10^{-4}\end{array}$ & 19664 & 4328 & 2478 & 4.078 & 1.002 & 0.530 \\
\hline$(0.43,0.57)$ & $\begin{array}{c}(0.1912,0.8088,138.7) \\
(0.2732,0.7268,79.62) \\
(0.43,0.57,52.14)\end{array}$ & $\begin{array}{c}-0.001 \\
0.0032 \\
0.0\end{array}$ & 15454 & 3516 & 2276 & 3.178 & 0.808 & 0.501 \\
\hline$(0.60,0.40)$ & $(0.60,0.40,43.69)$ & 0.0 & 10764 & 2554 & 1880 & 2.239 & 0.585 & 0.423 \\
\hline
\end{tabular}




\begin{tabular}{|c|c|c|c|c|c|c|c|c|c|c|}
\hline \multirow{3}{*}{ 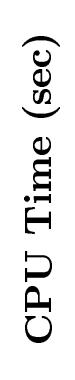 } & \multirow{3}{*}{ 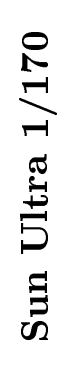 } & $\begin{array}{l}\sum_{2} \\
U_{1} \\
I_{1}\end{array}$ & \multicolumn{3}{|l|}{ 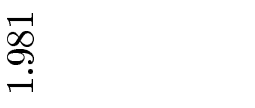 } & \multicolumn{3}{|l|}{$\begin{array}{l}\text { No } \\
\text { O } \\
\stackrel{+}{*}\end{array}$} & 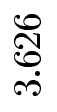 & $\begin{array}{l}\infty \\
\stackrel{0}{0} \\
\stackrel{0}{0}\end{array}$ \\
\hline & & $\begin{array}{l}D_{2} \\
U_{E}\end{array}$ & \multicolumn{3}{|c|}{ 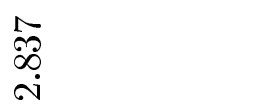 } & \multicolumn{3}{|l|}{ 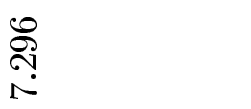 } & 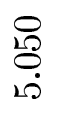 & $\begin{array}{l}\hat{0} \\
\infty \\
0 \\
0\end{array}$ \\
\hline & & E & \multicolumn{3}{|l|}{ 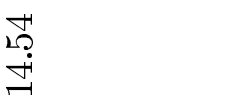 } & \multicolumn{3}{|l|}{$\begin{array}{l}0 \\
\sim \\
\stackrel{\sim}{*}\end{array}$} & $\stackrel{10}{\rightleftarrows}$ & 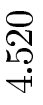 \\
\hline \multirow{3}{*}{ 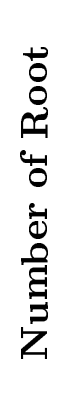 } & \multirow{3}{*}{ 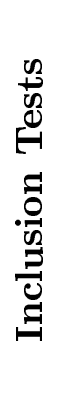 } & $\begin{array}{l}\sum_{2} \\
\text { D } \\
U_{1}\end{array}$ & \multicolumn{3}{|l|}{ 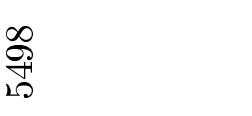 } & \multicolumn{3}{|l|}{$\underset{\stackrel{N}{\sim}}{\stackrel{\overbrace{}}{-1}}$} & \begin{tabular}{l}
\multirow{2}{\hat{O}}{} \\
Oे
\end{tabular} & $\frac{\vec{H}}{\stackrel{\Delta}{S}}$ \\
\hline & & $U_{1}^{\infty}$ & \multicolumn{3}{|l|}{ 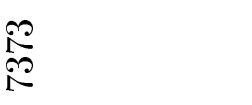 } & \multicolumn{3}{|l|}{$\begin{array}{l}\infty \\
\infty \\
\stackrel{\infty}{\infty} \\
\infty \\
\infty\end{array}$} & 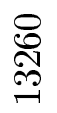 & 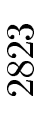 \\
\hline & & E & \multicolumn{3}{|l|}{$\begin{array}{l}\infty \\
\stackrel{0}{0} \\
\stackrel{2}{\circ}\end{array}$} & \multicolumn{3}{|l|}{$\begin{array}{l}\stackrel{N}{\oplus} \\
\stackrel{\infty}{\circ} \\
\stackrel{\Xi}{\sigma}\end{array}$} & 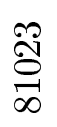 & 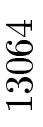 \\
\hline & ค & & $\begin{array}{l}0 \\
1 \\
0 \\
\vec{x} \\
\infty \\
\infty \\
10 \\
i \\
i\end{array}$ & $\stackrel{\circ}{\circ}$ & $\begin{array}{l}\stackrel{\infty}{+}+1 \\
\stackrel{+}{0} \\
\stackrel{0}{1}\end{array}$ & $\stackrel{0}{0}$ & 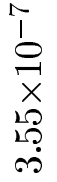 & 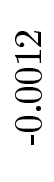 & $\stackrel{0}{0}$ & $\stackrel{0}{0}$ \\
\hline & $\begin{array}{l}\frac{n}{0} \\
\stackrel{0}{0} \\
0 \\
0\end{array}$ & 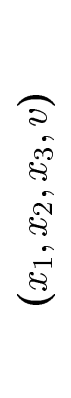 & 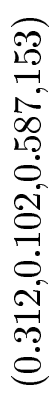 & 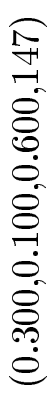 & $\begin{array}{l}0 \\
10 \\
\stackrel{1}{1} \\
0 \\
0 \\
0 \\
0 \\
0 \\
0 \\
0 \\
0 \\
0 \\
0 \\
0 \\
0 \\
0 \\
0\end{array}$ & 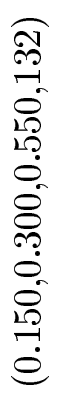 & 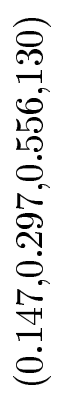 & 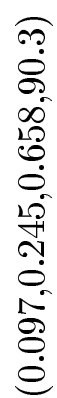 & 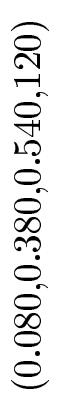 & 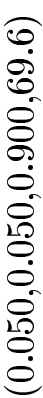 \\
\hline & D & 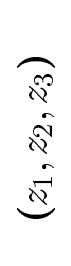 & $\begin{array}{l}\overparen{8} \\
\dot{0} \\
0 \\
0 \\
0 \\
\stackrel{0}{0} \\
\dot{0} \\
\dot{0} \\
\stackrel{0}{0}\end{array}$ & & & $\begin{array}{l}0 \\
10 \\
0 \\
0 \\
0 \\
0 \\
0 \\
0 \\
10 \\
\stackrel{0}{0} \\
\stackrel{0}{0}\end{array}$ & & & $\begin{array}{l}\overparen{F} \\
\stackrel{5}{0} \\
0 \\
\infty \\
\infty \\
0 \\
0 \\
\infty \\
\infty \\
0 \\
\dot{0}\end{array}$ & $\begin{array}{l}\overparen{8} \\
\dot{0} \\
\dot{0} \\
\dot{8} \\
\dot{0} \\
\dot{0} \\
\dot{0} \\
\dot{0}\end{array}$ \\
\hline
\end{tabular}

